\title{
The cellular basis of dendrite pathology in neurodegenerative diseases
}

\author{
Jung Hyun Kweon ${ }^{1, \#}$, Sunhong Kim ${ }^{2,3, \#} \mathcal{E}$ Sung Bae Lee ${ }^{1, *}$ \\ ${ }^{1}$ Department of Brain \& Cognitive Sciences, DGIST, Daegu 42988, ${ }^{2}$ Disease Target Structure Research Center, Korea Research Institute of \\ Bioscience and Biotechnology (KRIBB), Daejeon 34141, ${ }^{3}$ Department of Biomolecular Science, University of Science and Technology, \\ Daejeon 34141, Korea
}

\begin{abstract}
One of the characteristics of the neurons that distinguishes them from other cells is their complex and polarized structure consisting of dendrites, cell body, and axon. The complexity and diversity of dendrites are particularly well recognized, and accumulating evidences suggest that the alterations in the dendrite structure are associated with many neurodegenerative diseases. Given the importance of the proper dendritic structures for neuronal functions, the dendrite pathology appears to have crucial contribution to the pathogenesis of neurodegenerative diseases. Nonetheless, the cellular and molecular basis of dendritic changes in the neurodegenerative diseases remains largely elusive. Previous studies in normal condition have revealed that several cellular components, such as local cytoskeletal structures and organelles located locally in dendrites, play crucial roles in dendrite growth. By reviewing what has been unveiled to date regarding dendrite growth in terms of these local cellular components, we aim to provide an insight to categorize the potential cellular basis that can be applied to the dendrite pathology manifested in many neurodegenerative diseases. [BMB Reports 2017; 50(1): 5-11]
\end{abstract}

\section{INTRODUCTION}

Neurodegenerative diseases include a range of neuronal disabilities such as Alzheimer's disease (AD), Parkinson's disease (PD), Huntington's disease (HD), and Lou Gehrig's disease/Amyotrophic lateral sclerosis (ALS) (1). Although the manifestation of the symptoms and the progress of these diseases differ, there exist certain features which are common to many diseases. One such feature is the accumulation of

${ }^{*}$ Corresponding author. Tel: +82-53-785-6122; Fax: +82-53-7856109; E-mail: sblee@dgist.ac.kr

${ }^{\#}$ These authors contributed equally to this work.

https://doi.org/10.5483/BMBRep.2017.50.1.131

Received 1 August 2016

Keywords: Cytoskeleton, Dendrite pathology, Golgi outposts, Mitochondria, Neurodegenerative diseases toxic proteins (1). The other common feature is the massive neuronal loss in the brain of the late-onset patients, which renders their name "neurodegenerative diseases"(2), although the affected brain regions vary depending on the types of the diseases. Notably, this massive neuronal cell death is associated primarily with the late stages of the diseases, and may not account for the disease symptoms at the early stages. For example, the brains of polyglutamine (polyQ) disease patients typically show eventual neuronal loss 10-20 years after onset of symptoms (3). Consistent with this, it has been observed that the animal models of neurodegenerative diseases, such as HD (4) and PD $(5,6)$, displayed behavioral symptoms even without severe neuronal loss. In other words, these findings indicate that certain neuronal alterations in function and/or morphology preceding cell death contribute crucially to the initiation of symptoms at the early stages of neurodegenerative diseases.

As a plausible candidate of these neuronal alterations preceding cell death, dendrite pathology in AD (7), PD (8), polyQ diseases $(9,10)$, and ALS (11) have been well recognized in both animal models and human patients. However, the exact nature of the dendrite pathology in these neurodegenerative diseases still remains largely elusive. From extensive studies done so far, we are now aware that the different diseases are associated with different patterns of dendritic spine and dendrite destabilization (12). It is notable that the dendrite pathology at the early stages of the diseases is phenotypically distinguishable from the dendrite degeneration of dying neurons. Dying neurons display dendrite blebbing/ beading, followed by extensive cleavages of dendrites involving rapid disruption of dendritic cytoskeletons shortly after the turning-on of the death signal [e.g. under hypoxic condition (13)]. On the other hand, the dendrites affected by neurodegenerative diseases at the early stage tend to undergo relatively slow and progressive alterations, or maintain the changed shape for a while [e.g. polyQ diseases, (9)].

The molecular control of dendrite growth seems very complex and involves a large number of regulator molecules performing diverse molecular functions such as transcriptional control, RNA metabolism, signal transduction, and so on (14). Since numerous molecules are either directly or indirectly

ISSN: 1976-670X (electronic edition)

Copyright (C) 2017 by the The Korean Society for Biochemistry and Molecular Biology

(c) This is an open-access article distributed under the terms of the Creative Commons Attribution Non-Commercial License (http://creativecommons.org/licenses/by-nc/4.0) which permits unrestricted non-commercial use, distribution, and reproduction in any medium, provided the original work is properly cited. 
associated with the pathogenesis of neurodegenerative diseases, when we consider a possible molecular mechanism underlying each case of dendrite defects in various neurodegenerative diseases, there are numerous combinations of these molecules regulating the dendrite growth. On the other hand, only a small number of cellular components are closely associated with dendrite growth. These cellular components, such as cytoskeletal structures, are supposed to serve as a final effector of dendrite growth at the downstream of the complex signaling from the aforementioned numerous molecules associated with the dendrite growth. Importantly, emerging evidence suggests that local cellular components located within dendrites play very crucial roles in the control of their growth (14). These local cellular components include dendritic cytoskeletal structures consisting of F-actin and microtubules, Golgi outposts (GOPs, local Golgi apparatus in dendrites), and dendritic mitochondria (Fig. 1). To our knowledge, the dendrite defects appear to be the outcome of combinatorial alterations in these local cellular components. Although the defects in the same type of cellular components (e.g. defects in dendritic F-actin) can result in variable dendrite phenotypes

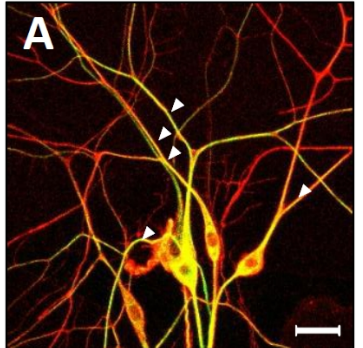

Microtubule

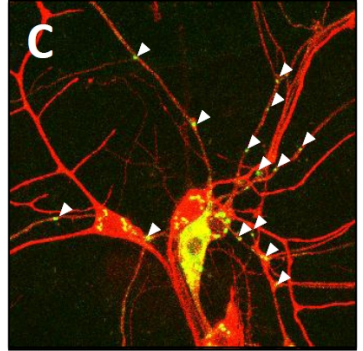

Golgi outposts

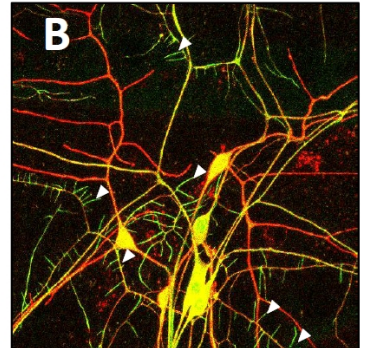

F-actin

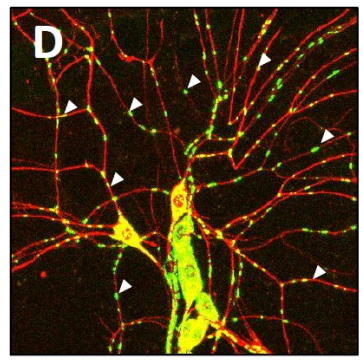

Mitochondria
Fig. 1. Localization of dendritic cellular components in Drosophila melanogaster dorsal cluster dendritic arborization (da) neurons. Merged images of local cellular components (green, marked by arrowheads) with membrane marker protein, CD4-tdTOM (red). Dendritic distribution of microtubules labeled by Tau-GFP (A), F-actin labeled by GMA (B), GOPs labeled by galT-eGFP (C), and mitochondria labeled by Mito-GFP (D), was examined in dorsal cluster da neurons by using 109(2)80-Gal4 driver. Dendrite images of da neurons of Drosophila 3rd instar larva located in abdominal segments A2 to A4 were captured using confocal microscopy. Scale bar indicates $50 \mu \mathrm{m}$. (e.g. dendrite branching defect harboring much less number of branches or terminal dendrite shortening), the altered cellular component from these known or predictable phenotypic variations in dendrites can be easily predicted. This article reviews how local cellular players affect the dendrite growth in both normal and pathological conditions, and we hope to establish clear criteria for categorizing types of dendrite pathology manifested in a number of neurodegenerative diseases. In the following section, we will focus on three local cellular components [dendritic cytoskeleton as a structural backbone, GOPs as a local supplier of plasma membrane (PM), and dendritic mitochondria as a local supplier of $\mathrm{Ca}^{2+}$ and ATP] (Fig. 2) closely associated with dendrite growth.

\section{DISTURBANCE OF LOCAL CELLULAR COMPONENTS TO BE RESPONSIBLE FOR THE DENDRITE PATHOLOGY IN NEURODEGENERATIVE DISEASES}

\section{Dendritic changes resulted from cytoskeletal alterations in} dendrites

There are two major cytoskeletal components within cells. They are filamentous actin (F-actin) and microtubules (15). To perform diverse biological activities, cells constantly undergo dynamic assembly and disassembly of these cytoskeletons under tight control of upstream regulators. The cytoskeletons serve as a backbone of cells that primarily supports the cellular structures, and are also involved in the transport of intracellular cargoes. In highly polarized neuron cells, the relative composition of these two components in dendrites is a crucial factor determining the dendrite shapes (12). In general, the main dendrite branches are known to be primarily supported by a packed network of microtubules, whereas the terminal dendrites (such as spines and filopodia) are mainly supported by F-actin structures (12). A previous study intriguingly proposed the exception to this general rule that microtubules are also present in spines, and may play an important role in

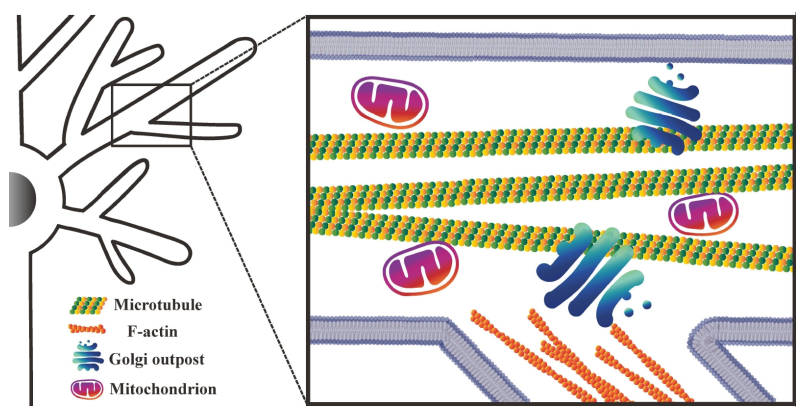

Fig. 2. A schematic diagram showing local cellular components crucial for dendrite growth. Microtubules are located mostly in the primary branches, and F-actin is located in both primary branches and terminal dendrites (the diagram depicts F-actin at terminal dendrites only). Mitochondria and GOPs are distributed throughout the dendrites. 
the control of spine development (16). After complete establishment of the dendritic field, the main dendrite branch (supported primarily by microtubules) remains relatively static at some homeostatic 'set point' in dendrite size (12), while the terminal dendrite (mainly consisting of F-actin) continuously undergoes dynamic changes (17-20).

Given the role of dendritic cytoskeletons as a structural backbone of highly polarized neurons, it is easy to conceive that alterations in these dendritic cytoskeleton result in dendritic changes. The best example of microtubule-mediated dendritic changes during normal development, comes from studies on the dendrite remodeling using a Drosophila sensory neuronal system (21). These studies successfully identified the severing of microtubule structures by local caspases as a key step in the remodeling process of dendrites known as dendrite pruning $(21,22)$. Another example potentially links microtubules to dendrite growth in normal conditions; a recent study reported that the microtubule array near a dendritic branch site serves as a docking site for a GOP, another crucial regulator of dendrite growth, which potentially links the microtubule organization to the regulation of dendrite growth (23). The question remains whether there is any evidence linking alterations in microtubules to dendrite pathology in neurodegenerative diseases. Firstly, the changes in microtubule dynamics have been reported in many neurodegenerative diseases (24). A representative example is the change in the phosphorylation status of tau, a microtubule-binding protein, leading to the formation of neurofibrillary tangle in $A D(25)$. In addition, microtubule depolymerization has been proposed as crucial for PD pathogenesis (26). Other direct evidences link microtubules to dendrite pathology. For example, LRRK2/Park8 mutation is known to induce dendrite degeneration involving microtubule fragmentation together with tau (27). Moreover, the alterations in dendritic microtubule dynamics have been reported to be linked to the dendrite pathology of polyQ diseases (28).

As for F-actin, several key players in the actin regulatory machinery such as Rac1, RhoA, and their associated signaling pathways, have been extensively studied for their roles in dynamic control of terminal dendrite growth (29). For tight control of F-actin formation, numerous regulator molecules of F-actin act on multiple processes related to F-actin, such as capping, severing, nucleation, and crosslinking/bundling, and so on (30). According to the previous studies, over $80 \%$ of the dendritic F-actin undergoes turn-over every minute, whereas $75 \%$ of dendritic microtubules undergo turn-over within tens of minutes (12). This means that F-actin is dynamically better controlled than microtubules, and thus neurons may be more sensitive to temporal or chronic changes in the regulator activity of F-actin in shaping terminal dendrites after the full establishment of dendritic arbors. In line with this, the pathological implication of F-actin defect was investigated in the context of many neurodegenerative diseases, including a subset of polyQ diseases, PD, and AD. Our previous study characterized that the toxicity from a subset of nuclear polyQ proteins, such as pathogenic forms of SCA3/MJD1 and SCA1, cause almost complete ablation of F-actin structures in dendrites in Drosophila sensory neurons (9). Interestingly, these F-actin alterations lead to cell type-specific terminal dendritic defects due to different compositions of cytoskeletal make-ups of dendrites in the different types of neurons. In addition, the LRRK2/Park8 function has been linked to the regulation of actin dynamics (31). Moreover, abnormal bundling and accumulation of F-actin were reported in the tau-induced AD model (32). In AD brain, aggregates of ADF/Cofilin have also been described in association with both amyloid plaques and neurofibrillary tangles $(33,34)$. Despite these extensive studies, further studies are required to clearly link F-actin defects to specific dendrite pathology in neurodegenerative diseases.

\section{Dendritic changes derived from alterations of GOPs}

Neurons have a vast PM area due to their complex structure. For example, it has been estimated that neurites may have about 30 times greater surface area than the surface area of a soma in cultured hippocampal neurons (35). This implies that highly polarized neurons should have an active machinery performing PM supply and recycling, compared to other cells. Especially, the terminal structures of neurons, such as dendrite terminals, need a constant and very active membrane supply due to their nature of undergoing dynamic changes in morphology (36). Indeed, neurons are known to have their unique local systems for membrane supply and recycling to fulfill this local need (37).

In general, Golgi apparatus receives materials from ER, and plays a key role in the secretory pathway that sends vesicles intra-cellularly and supplies PM through exocytosis, regardless of cell types. In neurons, a specialized local Golgi structure, GOP, is present in dendrites $(38,39)$. According to a recent study, GOP ablation inhibits the dendritic growth (40). In spite of these seminal works, details about GOPs remain largely elusive. For example, the exact differences in their nature between somatic Golgi and GOPs is unknown. Also, the source of GOPs remain controversial, whether somatic Golgi fragment is transported to distal dendrites to form GOPs or local ER elements generate the GOPs (42).

To date, GOPs have been implicated with a few neurodegenerative diseases. Interestingly, a recent study showed that Lrrk2 mutation associated with PD is able to induce GOPmediated dendrite reduction (43). In addition, TDP43 mutation associated with ALS induces fragmentation of somatic Golgi (44), but the impact of somatic Golgi fragmentation on Golgi outposts is unidentified. However, a lot of unsolved questions still exist such as to how GOPs are affected by these disease conditions.

Alteration in mitochondrial physiology affecting dendrites Mitochondria are critical for supplying cellular energy and buffering $\mathrm{Ca}^{2+}$. In the neuronal system, these functions of 
mitochondria are more crucial than in other cells because of the following reasons. First, neurons have elongated and complex structures, therefore the simple diffusion of ATP produced from somatic mitochondria is difficult and not sufficient to fulfill the local need (45). Second, neuronal firing is accompanied by massive influx of $\mathrm{Ca}^{2+}$, hence the ability of mitochondria to buffer $\mathrm{Ca}^{2+}$ is particularly important in neurons (46).

These functions of mitochondria are crucial for the dendrite growth. For example, adequate supply of ATP by mitochondria is very essential for F-actin elongation associated with dendrite growth (47). In addition, $\mathrm{Ca}^{2+}$ signaling has been implicated in the regulation of dendritic development (48). High amount of $\mathrm{Ca}^{2+}$ is known to result in excitotoxicity, inducing dendritic degeneration (49). Notably, there are a couple of papers regarding changes in mitochondrial physiology linked to dendrite phenotypes: mitochondrial fusion/fission status has been linked to dendrite phenotypes (50), and a mutation in a specific mitochondrial protein, preli-like, is known to induce dendritic changes (51). However, the exact mechanism of how mitochondria affect dendrite morphology still remains unknown.

Notably, mitochondrial dysfunction has been reported in many neurodegenerative diseases (52). The changes in mitochondrial quality control system has been proposed as a key pathogenic mechanism of familial cases of PD (53). Thus, it will be interesting to see whether these mitochondrial changes are indeed linked to the dendrite pathology in these diseases.

\section{Drugs that have potential for amelioration of dendrite pathology}

In this section, we discuss the possible therapeutic strategies targeting the cytoskeleton in neurodegenerative diseases, and future targets. Microtubule-stabilizing compounds have been investigated and used as cancer therapeutics. Brain penetrant epothilone D (EpoD), which stabilizes microtubule similar to Taxol, has been demonstrated to rescue microtubule organization, cognitive defect, and tau pathology in a couple of mouse models $(54,55)$. Based on these results, a phase I clinical trial by Bristol-Myers Squibb was carried out from 2012 to 2013, but the results have not been announced so far (ClinicalTrials. gov identifier NCT01492374). In addition, EpoD rescued microtubule defects in PD mice model (56). Given that the balance of microtubule dynamics is important to maintain dendrite and axon integrity, the blood-brain barrier (BBB)permeable agents that can stabilize microtubule dynamics remain promising therapeutics for neurodegenerative diseases.

Microtubule dynamics is mainly regulated by microtubuleassociated proteins (MAPs). Tau and collapsin response mediator protein 2 (CRMP2), which belong to MAPs, are hyperphosphorylated in AD. GSK3 $\beta$ and CDK5 are the kinases responsible for the hyperphosphorylation $(57,58)$. The phosphorylation of tau and CRMP2 by CDK5 is a prerequisite for the subsequent phosphorylation by GSK3 $\beta$, which is called 'priming'. Both kinases are involved in neurodegenerative disorders $(58,59)$. Various compounds that block the activity of GSK3 $\beta$ have been investigated in neurodegeneration mice model and clinical trials. Tideglusib, a selective GSK3 $\beta$ inhibitor, showed not only decreased tau phosphorylation and improved behavioral functions, but also decreased $A \beta$ pathology and neuronal death in AD models $(60,61)$. However, a phase II trial using Tideglusib in $A D$ patients showed the drug safety in 26 weeks without clinical benefit (62). A recent work published this year demonstrated that DYRK1, a kinase overexpressed in Down's syndrome patients, can phosphorylate tubulin per se and inhibit their polymerization, leading to the defective dendrite patterning (63). It would be intriguing to see whether selective DYRK1 inhibitors modulate the disease symptoms of $\mathrm{AD}, \mathrm{PD}$, and ALS.

RhoA/ROCK pathway regulates the polymerization of actin cytoskeleton and has been implicated in AD, PD, HD and ALS (64). Although it is unclear whether the inhibition of this signaling is beneficial for the treatment of neurodegeneration, several evidences reveal that ROCK inhibitors may have potential to cure the diseases. For example, the knockdown of ROCK2 (an isoform strongly expressed in the brain) reduces the loss of dopaminergic neurons and improves motor behavior in 6-OHDA mice model (65). Several compounds selective for ROCK2 have been recently developed to treat autoimmune diseases. It would be of much interest to test the compounds in neurodegeneration mouse models. However, there is a concern to use ROCK inhibitors as a therapeutic for the neurodegenerative diseases. Recent work unveiled that RhoA ROCK pathway is required for GOP formation (66), hence the study of these inhibitors should be carefully interpreted.

Besides studying the specific targets for the diseases, several compounds that restore the ability of dendritic spine formation in neurons have been screened. One of the efforts led to the discovery of benzothiazole amphiphiles, which promote the formation of dendritic spines (67). These chemicals may facilitate spinogenesis through the activation of Ras pathway, although they are a bit toxic to the cells at their active concentration.

The drugs intervening cytoskeleton functions seem to have a huge potential in improving the symptoms in neurodegenerative diseases in mice models, but many obstacles still remain to be overcome. Target-related toxicity due to non-specific inhibition of cytoskeleton or kinases will be a great concern. In addition, the delivery of compounds to the central nervous system is always a tough question in drug discovery. Despite these circumstances, it is essential to make an effort to seek more selective and brain-penetrant therapeutics.

\section{CONCLUSION}

In this article, we reviewed alterations in the local structural supports by dendritic cytoskeletons, local supply of PM components by GOPs, and local supply of $\mathrm{Ca}^{2+}$ and ATP by dendritic mitochondria to be potentially associated with dendrite pathology in neurodegenerative diseases. As reviewed 
here, we begin to recognize the key players for decoding the mystery of the dendrite pathology, but many questions still remain to be addressed. We do not know the exact contribution of the dendrite pathology to the pathogenesis of the diseases and to the disease symptoms. Also, in particular, not much is known about the nature of GOPs. Thus, further studies are required to clearly address these questions. With the tremendous efforts of the related research fields, we hope to fully understand the cellular basis of the dendrite pathology manifested in many neurodegenerative diseases someday in the future, which will greatly help to develop effective treatments specifically targeting early stages of the diseases.

\section{ACKNOWLEDGEMENTS}

This work was supported by the DGIST R\&D and MIREBraiN program, Basic Science Research Program through the ministry of science, ICT \& future planning of Korea (16-BD-0402, 2013R1A1A1004978), and the Development of Platform Technology for Innovative Medical Measurements Program from the Korea Research Institute of Standards and Science (KRISS-2016-16011064) (S.B.L.).

\section{CONFLICTS OF INTEREST}

The authors have no conflicting financial interests.

\section{REFERENCES}

1. Forman MS, Trojanowski JQ and Lee VM (2004) Neurodegenerative diseases: a decade of discoveries paves the way for therapeutic breakthroughs. Nat Med 10, 10551063

2. Vila M and Przedborski S (2003) Targeting programmed cell death in neurodegenerative diseases. Nat Rev Neurosci $4,365-375$

3. Zoghbi HY and Orr HT (2000) Glutamine repeats and neurodegeneration. Annu Rev Neurosci 23, 217-247

4. Li X-J (1999) The early cellular pathology of Huntington's disease. Mol Neurobiol 20, 111-124

5. Goldberg MS, Fleming SM, Palacino JJ et al (2003) Parkin-deficient mice exhibit nigrostriatal deficits but not loss of dopaminergic neurons. J Biol Chem 278, 4362843635

6. Gispert S, Ricciardi F, Kurz A et al (2009) Parkinson phenotype in aged PINK1-deficient mice is accompanied by progressive mitochondrial dysfunction in absence of neurodegeneration. PLoS One 4, e5777

7. Baloyannis SJ (2009) Dendritic pathology in Alzheimer's disease. J Neurol Sci 283, 153-157

8. Villalba RM and Smith Y (2010) Striatal spine plasticity in Parkinson's disease. Front Neuroanat 4, 133

9. Lee SB, Bagley JA, Lee HY, Jan LY and Jan YN (2011) Pathogenic polyglutamine proteins cause dendrite defects associated with specific actin cytoskeletal alterations in Drosophila. Proc Natl Acad Sci U S A 108, 16795-16800

10. DiFiglia M, Sapp E, Chase KO et al (1997) Aggregation of huntingtin in neuronal intranuclear inclusions and dystrophic neurites in brain. Science 277, 1990-1993

11. Nakano I and Hirano A (1987) Atrophic cell processes of large motor neurons in the anterior horn in amyotrophic lateral sclerosis: observation with silver impregnation method. J Neuropathol Exp Neurol 46, 40-49

12. Koleske AJ (2013) Molecular mechanisms of dendrite stability. Nat Rev Neurosci 14, 536-550

13. Park JS, Bateman MC and Goldberg MP (1996) Rapid alterations in dendrite morphology during sublethal hypoxia or glutamate receptor activation. Neurobiol Dis 3, 215-227

14. Jan Y-N and Jan LY (2010) Branching out: mechanisms of dendritic arborization. Nat Rev Neurosci 11, 316-328

15. Fischer RS and Fowler VM (2015) Thematic Minireview Series: The State of the Cytoskeleton in 2015. J Biol Chem 290, 17133-17136

16. Gu J, Firestein BL and Zheng JQ (2008) Microtubules in dendritic spine development. J Neurosci 28, 12120-12124

17. Engert $F$ and Bonhoeffer $T$ (1999) Dendritic spine changes associated with hippocampal long-term synaptic plasticity. Nature 399, 66-70

18. Maletic-Savatic M, Malinow R and Svoboda K (1999) Rapid dendritic morphogenesis in CA1 hippocampal dendrites induced by synaptic activity. Science 283, 1923-1927

19. Toni N, Buchs PA, Nikonenko I, Bron CR and Muller D (1999) LTP promotes formation of multiple spine synapses between a single axon terminal and a dendrite. Nature 402, 421-425

20. Horch HW, Kruttgen A, Portbury SD and Katz LC (1999) Destabilization of cortical dendrites and spines by BDNF. Neuron 23, 353-364

21. Williams DW and Truman JW (2005) Cellular mechanisms of dendrite pruning in Drosophila: insights from in vivo time-lapse of remodeling dendritic arborizing sensory neurons. Development 132, 3631-3642

22. Kuo CT, Zhu S, Younger S, Jan LY and Jan YN (2006) Identification of E2/E3 ubiquitinating enzymes and caspase activity regulating Drosophila sensory neuron dendrite pruning. Neuron 51, 283-290

23. Ori-McKenney KM, Jan LY and Jan YN (2012) Golgi outposts shape dendrite morphology by functioning as sites of acentrosomal microtubule nucleation in neurons. Neuron 76, 921-930

24. Dubey J, Ratnakaran N and Koushika SP (2015) Neurodegeneration and microtubule dynamics: death by a thousand cuts. Front Cell Neurosci 9, 343

25. Zempel H and Mandelkow E (2014) Lost after translation: missorting of Tau protein and consequences for Alzheimer disease. Trends Neurosci 37, 721-732

26. Feng J (2006) Microtubule: a common target for parkin and Parkinson's disease toxins. Neuroscientist 12,469476

27. Lin $\mathrm{CH}$, Tsai PI, Wu RM and Chien CT (2010) LRRK2 G2019S mutation induces dendrite degeneration through mislocalization and phosphorylation of tau by recruiting autoactivated GSK3ss. J Neurosci 30, 13138-13149

28. Chen L, Stone MC, Tao J and Rolls MM (2012) Axon injury and stress trigger a microtubule-based neuropro- 
tective pathway. Proc Natl Acad Sci U S A 109, 1184211847

29. Hotulainen P and Hoogenraad CC (2010) Actin in dendritic spines: connecting dynamics to function. J Cell Biol 189, 619-629

30. Welch MD and Mullins RD (2002) Cellular control of actin nucleation. Annu Rev Cell Dev Biol 18, 247-288

31. Parisiadou $L$ and Cai $H$ (2010) LRRK2 function on actin and microtubule dynamics in Parkinson disease. Commun Integr Biol 3, 396-400

32. Fulga TA, Elson-Schwab I, Khurana V et al (2007) Abnormal bundling and accumulation of F-actin mediates tau-induced neuronal degeneration in vivo. Nat Cell Biol 9, 139-148

33. Minamide LS, Striegl AM, Boyle JA, Meberg PJ and Bamburg JR (2000) Neurodegenerative stimuli induce persistent $\mathrm{ADF} /$ cofilin-actin rods that disrupt distal neurite function. Nat Cell Biol 2, 628-636

34. Heredia L, Helguera P, de Olmos S et al (2006) Phosphorylation of actin-depolymerizing factor/cofilin by LIMkinase mediates amyloid beta-induced degeneration: a potential mechanism of neuronal dystrophy in Alzheimer's disease. J Neurosci 26, 6533-6542

35. Ye B, Zhang YW, Jan LY and Jan YN (2006) The secretory pathway and neuron polarization. J Neurosci 26, 10631-10632

36. Luscher C, Nicoll RA, Malenka RC and Muller D (2000) Synaptic plasticity and dynamic modulation of the postsynaptic membrane. Nat Neurosci 3, 545-550

37. Hanus C and Ehlers MD (2008) Secretory Outposts for the Local Processing of Membrane Cargo in Neuronal Dendrites. Traffic 9, 1437-1445

38. Horton AC and Ehlers MD (2003) Dual modes of endoplasmic reticulum-to-Golgi transport in dendrites revealed by live-cell imaging. J Neurosci 23, 6188-6199

39. Gardiol A, Racca C and Triller A (1999) Dendritic and postsynaptic protein synthetic machinery. J Neurosci 19, 168-179

40. Ye B, Zhang Y, Song $W$, Younger SH, Jan LY and Jan YN (2007) Growing dendrites and axons differ in their reliance on the secretory pathway. Cell 130, 717-729

41. Horton AC, Rácz B, Monson EE, Lin AL, Weinberg RJ and Ehlers MD (2005) Polarized secretory trafficking directs cargo for asymmetric dendrite growth and morphogenesis. Neuron 48, 757-771

42. Sengupta D and Linstedt AD (2011) Control of organelle size: the Golgi complex. Annu Rev Cell Dev Biol 27, 57-77

43. Lin $\mathrm{CH}$, Li H, Lee YN, Cheng YJ, Wu RM and Chien CT (2015) Lrrk regulates the dynamic profile of dendritic Golgi outposts through the golgin Lava lamp. J Cell Biol 210, 471-483

44. Fujita Y, Mizuno Y, Takatama M and Okamoto K (2008) Anterior horn cells with abnormal TDP-43 immunoreactivities show fragmentation of the Golgi apparatus in ALS. J Neurol Sci 269, 30-34

45. Hubley MJ, Locke BR and Moerland TS (1996) The effects of temperature, $\mathrm{pH}$, and magnesium on the diffusion coefficient of ATP in solutions of physiological ionic strength. Biochim Biophys Acta 1291, 115-121
46. Rizzuto R, De Stefani D, Raffaello A and Mammucari C (2012) Mitochondria as sensors and regulators of calcium signalling. Nat Rev Mol Cell Biol 13, 566-578

47. KoRN ED, Carlier M-F and Pantaloni D (1987) Actin polymerization and ATP hydrolysis. Science 238, 638-644

48. Redmond $L$ and Ghosh A (2005) Regulation of dendritic development by calcium signaling. Cell Calcium 37, 411-416

49. Greenwood SM and Connolly CN (2007) Dendritic and mitochondrial changes during glutamate excitotoxicity. Neuropharmacology 53, 891-898

50. Li Z, Okamoto K-I, Hayashi Y and Sheng M (2004) The importance of dendritic mitochondria in the morphogenesis and plasticity of spines and synapses. Cell 119, 873-887

51. Tsubouchi A, Tsuyama T, Fujioka M et al (2009) Mitochondrial protein Preli-like is required for development of dendritic arbors and prevents their regression in the Drosophila sensory nervous system. Development 136, 3757-3766

52. Burte F, Carelli V, Chinnery PF and Yu-Wai-Man P (2015) Disturbed mitochondrial dynamics and neurodegenerative disorders. Nat Rev Neurol 11, 11-24

53. Whitworth AJ and Pallanck LJ (2009) The PINK1/Parkin pathway: a mitochondrial quality control system? J Bioenerg Biomembr 41, 499-503

54. Barten DM, Fanara P, Andorfer C et al (2012) Hyperdynamic microtubules, cognitive deficits, and pathology are improved in tau transgenic mice with low doses of the microtubule-stabilizing agent BMS-241027. J Neurosci 32, 7137-7145

55. Zhang B, Carroll J, Trojanowski JQ et al (2012) The microtubule-stabilizing agent, epothilone $\mathrm{D}$, reduces axonal dysfunction, neurotoxicity, cognitive deficits, and Alzheimer-like pathology in an interventional study with aged tau transgenic mice. J Neurosci 32, 3601-3611

56. Cartelli D, Casagrande F, Busceti CL et al (2013) Microtubule alterations occur early in experimental parkinsonism and the microtubule stabilizer epothilone D is neuroprotective. Sci Rep 3, 1837

57. Tseng HC, Zhou Y, Shen Y and Tsai LH (2002) A survey of Cdk5 activator p35 and p25 levels in Alzheimer's disease brains. FEBS Lett 523, 58-62

58. Lei P, Ayton S, Bush Al and Adlard PA (2011) GSK-3 in Neurodegenerative Diseases. Int J Alzheimers Dis 2011, 189246

59. Cheung ZH and Ip NY (2012) Cdk5: a multifaceted kinase in neurodegenerative diseases. Trends Cell Biol 22, 169-175

60. Noh MY, Chun K, Kang BY et al (2013) Newly developed glycogen synthase kinase-3 (GSK-3) inhibitors protect neuronal cells death in amyloid-beta induced cell model and in a transgenic mouse model of Alzheimer's disease. Biochem Biophys Res Commun 435, 274-281

61. Sereno L, Coma M, Rodriguez M et al (2009) A novel GSK-3beta inhibitor reduces Alzheimer's pathology and rescues neuronal loss in vivo. Neurobiol Dis 35, 359-367

62. Lovestone S, Boada M, Dubois B et al (2015) A phase II trial of tideglusib in Alzheimer's disease. J Alzheimers Dis $45,75-88$

63. Ori-McKenney KM, McKenney RJ, Huang HH et al (2016) 
Phosphorylation of beta-Tubulin by the Down Syndrome Kinase, Minibrain/DYRK1a, Regulates Microtubule Dynamics and Dendrite Morphogenesis. Neuron 90, 551-563

64. Eira J, Silva CS, Sousa MM and Liz MA (2016) The cytoskeleton as a novel therapeutic target for old neurodegenerative disorders. Prog Neurobiol 141, 61-82

65. Saal KA, Koch JC, Tatenhorst L et al (2015) AAV.shRNAmediated downregulation of ROCK2 attenuates degeneration of dopaminergic neurons in toxin-induced models of Parkinson's disease in vitro and in vivo. Neurobiol Dis 73, 150-162

66. Quassollo G, Wojnacki J, Salas DA et al (2015) A RhoA Signaling Pathway Regulates Dendritic Golgi Outpost Formation. Curr Biol 25, 971-982

67. Cifelli JL, Dozier L, Chung T, Patrick GN and Yang J (2016) Benzothiazole Amphiphiles Promote the Formation of Dendritic Spines in Primary Hippocampal Neurons. J Biol Chem 291, 11981-11992 